\title{
A CONVENIENT SOLUTION TO GEOMETRIC DISTORTION AND ITS APPLICATION TO PHOEBE'S OBSERVATIONS
}

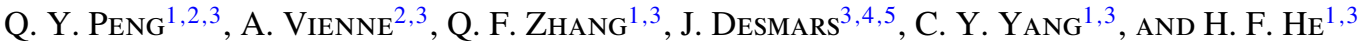 \\ ${ }^{1}$ Department of Computer Science, Jinan University, Guangzhou 510632, China; pengqy@ pub.guangzhou.gd.cn \\ ${ }^{2}$ University of Lille 1, LAL-IMCCE UMR 8028, F-59000 Lille, France \\ ${ }^{3}$ Sino-French Joint Laboratory for Astrometry, Dynamics and Space Science, Jinan University, Guangzhou 510632, China \\ ${ }^{4}$ Shanghai Astronomical Observatory, Chinese Academy of Sciences, Shanghai 200030, China \\ 5 IMCCE-Observatoire de Paris, UMR8028 du CNRS, UPMC, 77 Avenue Denfert-Rochereau, F-75014 Paris, France \\ Received 2012 April 5; accepted 2012 September 27; published 2012 November 8
}

\begin{abstract}
A simple but effective approach is proposed for measuring the geometric distortion of a CCD field of view of a ground-based telescope. For three open clusters (M35, M67, and NGC 2324), 425 CCD frames taken by a $1 \mathrm{~m}$ telescope at the Yunnan Observatory are used to test this approach. It is found that the geometric distortion pattern depends strongly on the corresponding filter used. The geometric distortion is then used to correct the pixel positions for Phoebe, the ninth satellite of Saturn, and its reference stars imaged in 220 CCD frames taken by the same telescope. The standard deviation of the $(\mathrm{O}-\mathrm{C}$; observed minus computed $)$ residuals of Phoebe is significantly improved after correcting the geometric distortions.
\end{abstract}

Key words: astrometry - planets and satellites: individual (Phoebe) - techniques: image processing

\section{INTRODUCTION}

At the end of 2008, the CCD camera (with a resolution of $1024 \times 1024$ with $24 \mu \mathrm{m}$ for each pixel) of the $1 \mathrm{~m}$ telescope at Yunnan Observatory was updated to a slightly larger CCD camera with a resolution of $2048 \times 2048(13.5 \mu \mathrm{m}$ for each pixel). In order to check the astrometric property of the new receiver, we try to solve its geometric distortion so that astrometric observations of some planetary satellites can be continued. It is well known that in the astrometric CCD observations of planetary satellites (such as the major satellites of Saturn), the two parameters of the scale factor and orientation of the CCD chip have long been supposed to be without any distortion and accurate enough to calibrate its field of view (Colas \& Arlot 1991; Harper et al. 1997; Shen et al. 2001; Vienne et al. 2001; Peng et al. 2012). In addition, the stability of two parameters has also been taken for granted. The key reason for these suppositions is the small CCD field of view. However, a small CCD field of view may not always be qualified for highprecision astrometry. An obvious example is the astrometry for the Hubble Space Telescope (HST). A very small field of view of $80^{\prime \prime} \times 80^{\prime \prime}$ for each WF chip (for its WFPC2 chips) has a maximum geometric distortion of about 5 pixels at the edge of its field (Anderson \& King 2003). Anderson \& King (2003) determined a more accurate solution for the geometric distortion (called GD hereafter) of HST's WFPC2 by increasing the accuracy of the linear terms. It is just after solving the GD that HST begins to tap its astrometric potential. For example, the observations of Saturnian satellites have much better precision than ever before (Poulet \& Sicardy 2001) mainly because of the GD correction according to French et al. (2006). Anderson et al. (2006) applied this experience with HST (Anderson \& King 2003) toward determining the GD of a ground-based telescope. They adopted a six-constant plate model for their ground-based telescope that implicitly removes atmospheric refraction.

In this paper, we propose an alternative approach, but take advantage of an astrometric catalog to derive our GD for a ground-based telescope. As a practical application, a series of CCD frames of Phoebe, the ninth satellite of Saturn, are used to test the solved GD and an obvious improvement in the precision of positional measurement is found.

In order to explain our approach, we recall our experience first from our ground-based astrometry. After a CCD frame for a target (such as Phoebe) was taken in a small field of view (for example, about $10^{\prime} \times 10^{\prime}$ or even smaller), two reference stars in the frame are usually the minimum requirement for our further reduction. This is because we do not know the accurate parameters of the telescope, the CCD chip, and the pointing beforehand (thus, these parameters must be approximate, like those in Table 1). In addition, these parameters sometimes/often change during a night observation. How then can we determine them?

A least-squares technique is the best choice for us. Specifically, we can first measure the pixel position $(x, y)$ of a reference star and the target by some centroid algorithm (such as a Gaussian fitting), and compute their respective standard coordinate $(\xi, \eta)$ (i.e., via central projection; see Green 1985 for more details) in terms of some assumed tangential point's equatorial coordinate (i.e., the pointing; $A, D$ ) and their own theoretical equatorial coordinates $(\alpha, \delta)$ by the following well-known formulae:

$$
\begin{aligned}
\xi & =\frac{\cos \delta \sin (\alpha-A)}{\sin \delta \sin D+\cos \delta \cos D \cos (\alpha-A)}, \\
\eta & =\frac{\sin \delta \cos D-\cos \delta \sin D \cos (\alpha-A)}{\sin \delta \sin D+\cos \delta \cos D \cos (\alpha-A)}
\end{aligned}
$$

Then only for the reference stars, we use a four-constant plate model (with an assumption that all astrometric effects are taken into account and the pixel of the CCD is square),

$$
\xi=a x-b y+c, \eta=b x+a y+d,
$$

where the equation simply describes a displacement, a rotation, and a scale factor between the standard coordinate on the tangential plane and the pixel coordinate on the CCD chip used. More exactly, $(c, d)$ is the offset, $a=\rho \cos \varphi$, and $b=\rho \sin \varphi$ in which $\rho$ and $\varphi$ are the scale factor of the telescope and the 
Table 1

Specifications of the Telescope and CCD Chip

\begin{tabular}{lc}
\hline \hline Approximate focal length & $13.3 \mathrm{~m}$ \\
$F$-ratio & 13 \\
Diameter of primary mirror & $100 \mathrm{~cm}$ \\
CCD field of view & $7^{\prime} .1 \times 7^{\prime} .1$ \\
Size of pixel & $13.5 \mu \times 13.5 \mu$ \\
Size of CCD array & $2048 \times 2048$ \\
Angular extent per pixel & $0^{\prime \prime} .21 \mathrm{pixel}^{-1}$ \\
\hline
\end{tabular}

orientation of the CCD chip with respect to the real equator, respectively. By a least-squares fitting, when more than two (or only two) reference stars are available, the solved parameters $(\hat{a}, \hat{b}, \hat{c}$, and $\hat{d})$ can be obtained. We can indirectly know the parameters of the telescope and the CCD chip.

Once the solved parameters are available, we use Equation (2) again but only for the target. Thus the observed standard coordinate $\left(\xi_{o}, \eta_{o}\right)$ of the target can be derived by using its measured pixel position $(x, y)$. After combining with a previously computed standard coordinate $\left(\xi_{c}, \eta_{c}\right)$, the positional residual (observed minus computed; $\mathrm{O}-\mathrm{C}$ ) of the target can be obtained as follows:

$$
\begin{gathered}
\Delta \xi=\xi_{o}-\xi_{c} \simeq \Delta \alpha \cos D, \\
\Delta \eta=\eta_{o}-\eta_{c} \simeq \Delta \delta .
\end{gathered}
$$

Now we can come to understand that the pointing error is not serious because of the difference of $(\Delta \xi, \Delta \eta)$ (we usually choose a star that is nearest to the center of the CCD frame as the tangential point, so there may be about one minute of degree for its error). On the one hand, the computed standard coordinate $\left(\xi_{c}, \eta_{c}\right)$ by central projection contains the effect of the pointing error; on the other hand, the observed standard coordinate $\left(\xi_{o}, \eta_{o}\right)$ contains the same effect of this error (the constant and rotational parts of the effect; see Green 1985 for more details) in Equation (2), therefore, the difference for this effect in Equation (3) can cancel out. In other words, for the constant offset and rotation part of the pointing error, our operation is accurate by the previous least-squares fitting. Therefore, we seldom mention the pointing error in our reduction. Actually, this is a classical practice.

Up to now, we have not taken the GD into account (in fact, we always assume that no GD exists owing to the small field of view. One can find some examples in our reference list such as Colas \& Arlot 1991, Harper et al. 1997, Vienne et al. 2001, Peng et al. 2004, 2012, and Peng \& Zhang 2006). A logical way is to remove the GD effect first from all original pixel positions of reference stars and the target in a CCD frame, and then perform the abovementioned classical reduction.

In order to derive the GD, our routine is somewhat different from the "relative astrometry" adopted by other researchers, but the key idea is the same. Our routine is based on classical practice and might be called "absolute astrometry," and thus it might be more easily understood. In principle, there are two coordinate systems, either a pixel coordinate system or an equatorial coordinate one, for deriving GD, but their effects should be equivalent. However, in order to demonstrate more clearly the physical meaning, the pixel coordinate system of the CCD is preferable. The key problem is the transformation of the residual form in right ascension like that in Equation (3), which is associated with the declination $(D)$ of the pointing.
The contents of this paper are arranged as follows. In Section 2, the principle of solving GD for a ground-based telescope is described. The observations used to derive GD and applied to the ninth satellite of Saturn (Phoebe) are explained in detail in Section 3. In Section 4, the results of the standard deviation (SD) solution and Phoebe are given. In addition, some discussion of our proposal for solving the GD are included in more detail in Section 5. Finally, some conclusions are drawn in Section 6.

\section{PRINCIPLE}

According to Anderson \& King (2003, p. 114), "a frame is distortion-free if the star positions that define it have been corrected in such a way that the positions of the same stars, measured in any image with a different pointing, but corrected in the same way, can be transformed into those of this frame with nothing more than a displacement, a rotation, and a scale factor." In other words, the star positions in one frame can be accurately associated with those of the same stars in another frame only by a four-constant plate model (in view of classical photography assuming square pixels and orthogonal axes). The distribution of GD is unique for a series of CCD frames, and depends only on the pixel coordinates (or location) of each CCD frame. In addition, any parameter in the four-constant plate model is not required to be constant for a series of CCD frames because we allow for changes in the focal length, the orientation of the CCD chip with respect to the real equator, and the telescope's pointing between any two CCD frames.

A direct method for deriving GD is to adopt an astrometric flat with an accurate astrometric catalog when a field of view associated with GD is used to observe a dense star field. Perhaps such an "astrometric flat field" is available for some areas (such as M92 observed by HST; see van der Marel et al. 2007), but it is not always convenient for all telescopes since the target celestial field is not always near the calibration target or even appearing in the observable sky. The future Gaia astrometric catalog may be the best choice. Anyway, at present, we have to resort to another technique for self-calibration.

Here, we present an alternative proposal. Similar to the practice by Anderson \& King (2003), we can take multiple dithered exposures of the same sky field at different offsets in a pattern of either "+" (see Figure 7 in Anderson et al. 2006) or “\#” (see Figure 2 in Bellini \& Bedin 2010). The latter pattern of "\#” should have a higher efficiency. In our experimental observations, the offsets between any two neighboring CCD frames are about $1^{\prime}$ in right ascension or in declination. Figure 1 shows a dithered observational scheme for CCD frames.

Anderson \& King (2003) and Anderson et al. (2006) use a "standard" overlapping plate method or "relative astrometry" to derive the GD, that is, they use the difference between the pixel location of a star in one image and the pixel location of the same star in other images. However, the method described in this work uses the difference between the observed pixel location of a star in an image and the calculated pixel location of that star indirectly from its catalog right ascension and declination coordinates, which can be called "absolute astrometry."

After all stellar images are automatically searched and their pixel positions extracted by a two-dimensional Gaussian fit technique, as many stellar images as possible are matched by our developed technique (Ren \& Peng 2010) to their corresponding stars in an astrometric catalog so that each matched star image has a unique identification (a strict requirement is not needed for its positional precision). Then we use the difference between 


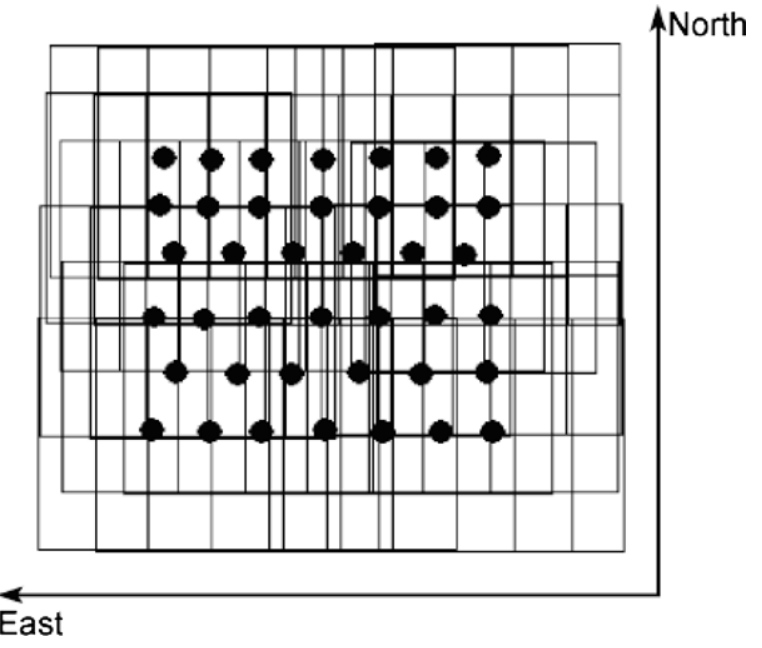

Figure 1. Dithered observational scheme for CCD frames. A box with a black spot in its center represents a CCD frame.

the observed pixel location $\left(x_{o}, y_{o}\right)$ of a star in an image and the pixel location $\left(x_{c}, y_{c}\right)$ of the same star indirectly calculated from its catalog equatorial coordinates $(\alpha, \delta)$ to derive the GD iteratively. Because the GD in an imaged pixel location and the catalog error $(\Delta \alpha, \Delta \delta)$ are entangled together, some operations have to be performed to disentangle them and eventually solve their effects accurately. A more detailed description is given as follows.

\subsection{Fundamentals in a CCD Frame}

For a star with an equatorial coordinate $(\alpha, \delta)$, its standard coordinate $(\xi, \eta)$ in the tangential plane can be computed according to Equation (1). Furthermore, we use a four-parameter linear transformation (instead of a four-constant plate model to distinguish from the previously described classical reduction) to describe approximately the relationship between the standard coordinate of a star and its measured pixel position. A detailed relationship is shown by the following equation:

$$
\begin{aligned}
& x=e \xi-f \eta+g, \\
& y=f \xi+e \eta+h,
\end{aligned}
$$

where $e=\cos \varphi / \rho$ and $f=-\sin \varphi / \rho$, in which $\rho$ and $\varphi$ are the scale factor and the orientation, respectively, of the CCD chip used. We should note that a four-parameter linear transformation is accurate only after all the astrometric effects (including the GD effect) are taken into account. Specifically, the atmospheric refraction effect (the standard model should be precise enough) is needed to add to the topocentric equatorial coordinates of a star or a target. In addition, the CCD chip must be square (or rectangular, but the scale ratio between its two axes has to be corrected strictly). Since the GD effect and the catalog error are unknown at this time, the transformation is approximate.

After Equation (4) is applied to all stars appearing in the same CCD frame, the estimates of the transformation parameters $\hat{e}, \hat{f}, \hat{g}$, and $\hat{h}$ can be derived by a least-squares fitting. They are not accurate at this time. Furthermore, the difference between the measured pixel location $\left(x_{o}, y_{o}\right)$ of a star and the indirectly calculated one $\left(x_{c}, y_{c}\right)$ of the same star using Equation (4) with the estimated parameters can be obtained as follows:

$$
\begin{aligned}
& \Delta x=x_{o}-x_{c}, \\
& \Delta y=y_{o}-y_{c} .
\end{aligned}
$$

Moreover, $(\Delta x, \Delta y)$ can be resolved into three components based on their respective error sources. First, the GD in the form of $(d x, d y)$ depends on only the imaged pixel location of the concerned star, and is independent of the astrometric catalog. In the second one, the catalog error $(\Delta \alpha, \Delta \delta)$ depends on only the referred catalog and is independent of the pixel position of the stellar image. The last component is the measured error $\left(v_{x}, v_{y}\right)$ that is related to the signal-to-noise ratio of the measured star. More exactly, we have the following expression:

$$
\begin{gathered}
\Delta x=d x+\hat{e} \Delta \alpha \cos D+v_{x}, \\
\Delta y=d y+\hat{e} \Delta \delta+v_{y},
\end{gathered}
$$

where the effect from the terms containing the parameter $f$ in Equation (4) is neglected since we can ensure that the $x$-axis of the CCD chip is nearly parallel to the real equator by a star-trailing operation before the observation of each night (i.e., $f \simeq 0$ ). In addition, only the first-order effect from the catalog error of the star on its pixel position is taken into account as far as a small field of view is concerned.

\subsection{An Important Relationship}

Furthermore, if a star is observed in two different CCD frames, noted as the $i$ th and the $j$ th, an important relationship can easily be derived as follows if the measured errors are temporarily neglected,

$$
\begin{gathered}
d x_{i}=\Delta x_{i}-\frac{\hat{e}_{i} \cos D_{i}}{\hat{e}_{j} \cos D_{j}} \Delta x_{j}+\frac{\hat{e}_{i} \cos D_{i}}{\hat{e}_{j} \cos D_{j}} d x_{j}, \\
d y_{i}=\Delta y_{i}-\frac{\hat{e}_{i}}{\hat{e}_{j}} \Delta y_{j}+\frac{\hat{e}_{i}}{\hat{e}_{j}} d y_{j},
\end{gathered}
$$

where all quantities with the suffix $i$ are associated with the $i$ th CCD frame and the suffix $j$ with the $j$ th CCD frame. It is shown in Equation (7) that the catalog error of the concerned star disappears since the same star and thus the same catalog error is canceled out during the derivation. This is an important relationship for the proposed approach.

A direct solution of the GD $\left(d x_{i}, d y_{i}\right)$ in the $i$ th CCD frame is impossible based only on two CCD frames because of the unknown distortion $\left(d x_{j}, d y_{j}\right)$ in the $j$ th CCD frame. However, if a star appears in $N(N \gg 2$, for example) CCD frames with different offsets, though they can be overlapped, for some definite frame noted as $i$ th, we have $(N-1)$ pairs of $\left(d x_{k}, d y_{k}\right)$ according to Equation (7) $(k=1,2, \ldots, N$, but $k \neq i)$ when the definite frame is associated, respectively, with every other frame. Obviously, the average of $(N-1)$ pairs of $\left(d x_{i}, d y_{i}\right)$, with each pair being connected to their corresponding $\left(d x_{k}, d y_{k}\right)$ using Equation (7), will embody to some degree the distortion $\left(d x_{i}, d y_{i}\right)$ at the pixel location $\left(x_{i}, y_{i}\right)$ of the concerned star in its definite frame because the $(N-1)$ pairs of distortion $\left(d x_{k}, d y_{k}\right)$ in $(N-1)$ different CCD frames can be viewed as random. In practice, we have many star images in overlapped CCD frames after a dense star cluster is observed. For each star, we can derive $N(N-1)$ pairs of $(d x, d y)$ (i.e., the GD) if the star appears in $N$ dithered CCD frames. Then we collect all pairs for all stars and divide these pairs into many equal-area boxes (such as $8 \times 8$ or $16 \times 16$ ) based on their original pixel locations. If a gradual variation is assumed for the GD distributions, the average in each box will be indicative of the GD at its center (called a grid point hereafter). Figure 2 shows us an example in which there 
Table 2

Observations for Open Cluster (Calibrated Fields) and Phoebe (Target)

\begin{tabular}{|c|c|c|c|c|c|}
\hline ObsDate & Cluster & $\begin{array}{l}\text { GD Solution } \\
\text { No. and Filter }\end{array}$ & $m$ & Reference Catalog & $\begin{array}{c}\text { Phoebe } \\
\text { No. and Filter }\end{array}$ \\
\hline 2011-02-24 & M35 & $54 \mathrm{I}$ & 242 & PPMXL (Roeser et al. 2010) & $10 \mathrm{I}$ \\
\hline $2011-02-26$ & NGC 2324 & $52 \mathrm{~N}$ & 318 & PPMXL (Roeser et al. 2010) & $21 \mathrm{~N}$ \\
\hline $2011-02-27$ & M35 & $43 \mathrm{I}$ & 264 & PPMXL (Roeser et al. 2010) & $15 \mathrm{I}$ \\
\hline 2011-04-01 & M67 & $47 \mathrm{I}$ & 103 & Yadav et al. (2008) & $19 \mathrm{I}$ \\
\hline 2011-04-03 & M67 & $26 \mathrm{R}, 32 \mathrm{I}$ & 118,128 & Yadav et al. (2008) & $23 \mathrm{R}, 55 \mathrm{I}$ \\
\hline 2011-04-04 & M67 & $28 \mathrm{R}, 25 \mathrm{I}$ & 118,137 & Yadav et al. (2008) & $30 \mathrm{I}$ \\
\hline Total & & 425 & & & 220 \\
\hline
\end{tabular}

Notes. Number and filter in Column 3 and the last column list the number of observed CCD frames and filter used for open clusters and Phoebe, respectively. Column 4 lists the mean number of stars in each CCD frame available to obtain the GD solution.

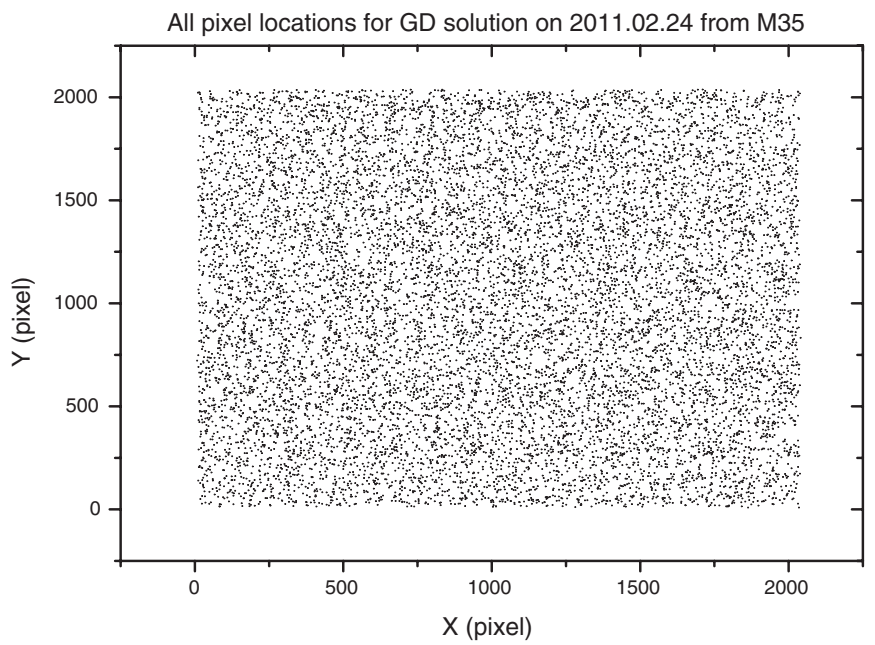

Figure 2. 13,061 pixel locations from 1009 matched stars (i.e., about 13 images per star on average) for the solution of GD. The observations were taken on the night of 2011 February 24 for the open cluster M35.

are about 200 star images on average in each box with a size of $256 \times 256$ square pixels. Actually, a $\sigma$-clipped criterion is applied to the actual processing. As such, the measured errors in Equation (6) will be highly compressed.

Although an initial GD distribution is derived, it is not precise since in the solution of our first four-parameter linear transformation using Equation (4), the observed pixel position of the star concerned contains the GD effect and catalog error, thus making the parameters $(\hat{e} \sim \hat{h})$ inaccurate. Obviously, an iterative procedure is indispensable.

\subsection{Iterative Solution of $G D$}

After our derivation of the initial GD in all grid points of a CCD frame, a precise solution of GD can be achieved simply by using an iteration procedure. The pixel position of every star image can be corrected from our initial GD by some interpolation technique. The iterative solution can be performed again. After this step, a differentiated GD correction is added to the initial GD to become a new GD distribution. This operation can be performed repeatedly until the absolute value of the differentiated GD correction in each grid point and in each coordinate direction is less than some preset threshold (0.01 pixel, for example). We find from our computation that five to eight iterations are usually enough to have a converged GD solution for the preset threshold of 0.01 pixel.

It seems that the catalog error may have some effect on the GD solution. However, it is usually random compared to the GD distribution. Therefore, on the whole the catalog error has a negligible effect on the GD solution.

The GD derived by the operations above is certainly a general and mean distribution for all CCD frames used. Different from the presentation of the GD by Anderson \& King (2003), where a third-order polynomial was fitted and some coefficients were designed to be zero because of an unknown absolute scale factor, we prefer in its application to let the GD remain in its original and numerical presentation and never to perform any further fit to its analytical form. That is to say, when a pixel location in a CCD frame is needed to remove its GD effect, a bilinear interpolation is made from the GD values of its four neighboring grid points among the GD distribution (like those in Table 3). This is the same operation that we perform to solve the GD distribution. The advantage of this algorithm is that we do not lose any precision.

\section{OBSERVATIONS}

Two observational campaigns were performed during the first half of 2011. In each campaign, four-night observations were captured by a $1 \mathrm{~m}$ telescope at the Yunnan Observatory (longitude-E102 $47^{\prime} 18^{\prime \prime}$; latitude-N25 $1^{\prime} 30^{\prime \prime}$; and height $-2000 \mathrm{~m}$ above sea level). Table 1 lists the specifications for the telescope and its CCD chip (DW436 of Andor Inc.). Detailed observations are listed in Table 2. Three open clusters (M35, M67, and NGC 2324) were observed in order to solve the GD of the field of view. In total, there were 425 CCD frames captured based on two filters (Johnson $I$ and $R$ ) and a null filter (noted as $N$ in Table 2). In each night, Phoebe, the ninth satellite of Saturn, was observed but in different pointings from the calibrated area. In total, 220 CCD frames were taken for Phoebe. For the CCD frames of Phoebe, usually only a few (sometimes only two) UCAC2 (Zacharias et al. 2004) reference stars appear in the field of view. Obviously, a complete solution of the GD for its field of view is impossible. We adopted the solved mean GD from open clusters with the same filter and in the same night to correct the GD effects for the pixel positions of Phoebe and its reference stars in UCAC2. There is one exception for the observations on February 25, when the GD without a filter is adopted from that on February 26 (see Table 2). 
Feb 25 Max=1.02 Med=0.39

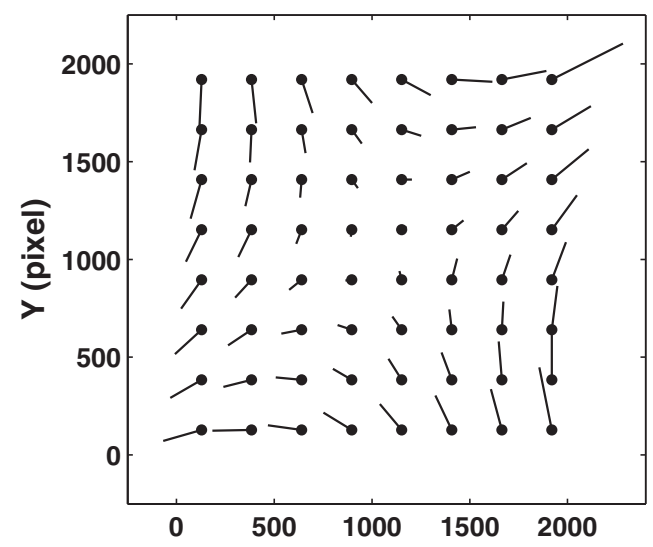

Apr 03 Max $=0.78 \mathrm{Med}=0.34$

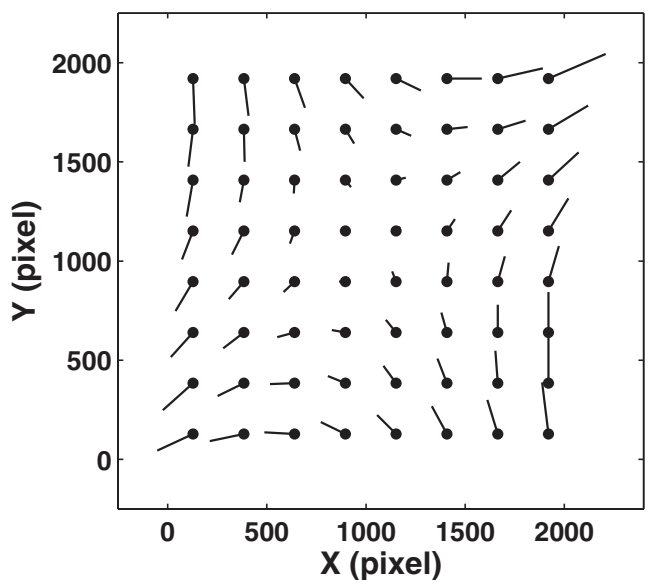

Apr 02 Max $=0.87$ Med $=0.38$
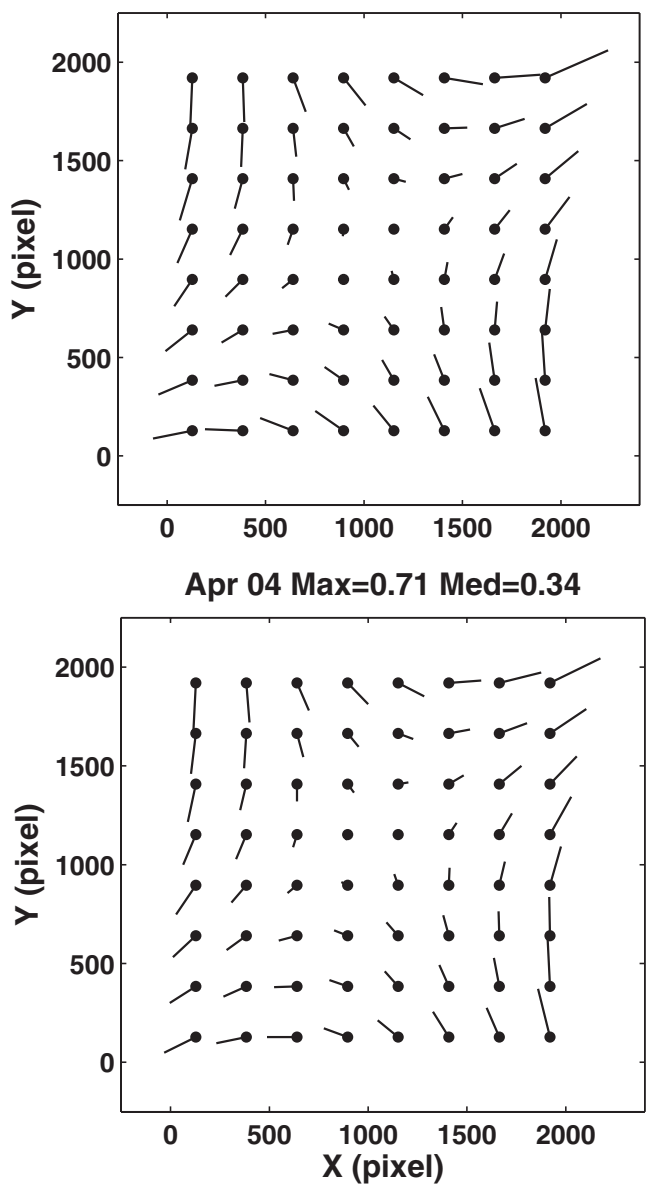

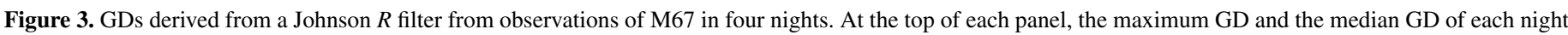
are also listed in units of pixels. In these panels, a factor of 400 is used to exaggerate the magnitude of each GD vector.

\section{RESULTS}

\subsection{GD and Filter Used}

When the same filter is used on different nights to derive the pattern of GD, it is found to be similar. Figure 3 shows all GD distributions when a Johnson $R$ filter is used and the open cluster M67 (i.e., NGC 2682) is observed on four different nights. The maximum GD changes from 0.71 pixels $(\sim 148$ mas) on April 4 to 1.02 pixels ( $\sim 213$ mas) on February 25 , but with a similar median of about 0.36 pixels ( $\sim 75$ mas; a factor of 400 is used to exaggerate each vector in this figure and in Figures 4 and 5 as well). While a Johnson $I$ filter is replaced in other night observations, similar (but with less magnitude) distributions of GD also appeared (see Figure 4). However, the GD distribution derived from the Johnson $I$ filter is significantly different from the distribution in the Johnson $R$ filter. We speculate that the difference may be caused by the inhomogeneity in the thickness of the filter used.

Although the similarity of the GD distributions exists when the same filter is used, the variations on two different nights cannot be neglected. Table 3 shows the mean GD and its corresponding standard deviation (called SD hereafter) on several nights. We find that most SDs are less than 0.05 pixels ( $\sim 10$ mas) in each direction, but a standard deviation as great as 0.12 pixels ( $\sim 25$ mas) also exists, which is not negligible when compared with the positional errors of reasonable bright stars (see Figure 7). The reasons for this may stem from the stability of the telescope in mechanics and optics, and/ or from environmental conditions such as temperature and pressure. More exactly, the plate constants depend on factors like temperature and are affected by others like telescope flexure. These factors can change from night to night and, in the case of flexure, on the region pointed at in the sky. In addition, the CCD can be dismounted and remounted in a different orientation between one night and the next (as described by Harper et al. 1997). Further study for these reasons is obviously needed in future work. It is surprising that even when no filter is used the GD distribution is obvious enough and reveals a swirllike structure (see Figure 5). This might also be caused by the inhomogeneity in the thickness of the front glass of the CCD window. Perhaps because the distance between the front glass of the CCD window and the CCD chip is less than that between a filter and the chip, or/and the better flatness of the front glass, the magnitude of the GD becomes less (see, for example, van Altena \& Monnier 1968 for more details). Further investigation is interesting and is needed in the future.

\section{2. (O - C) Residuals for Open Clusters}

Some main results for open clusters are given here. As an example, Figure 6 shows the positional $(\mathrm{O}-\mathrm{C})$ residuals of M35 observed on February 24 and 27, respectively, for the common stars. Good agreement can be found. Figure 7 shows the SD of the same stars in the open cluster M35 before and after GD correction. It is clear that the SD of the 

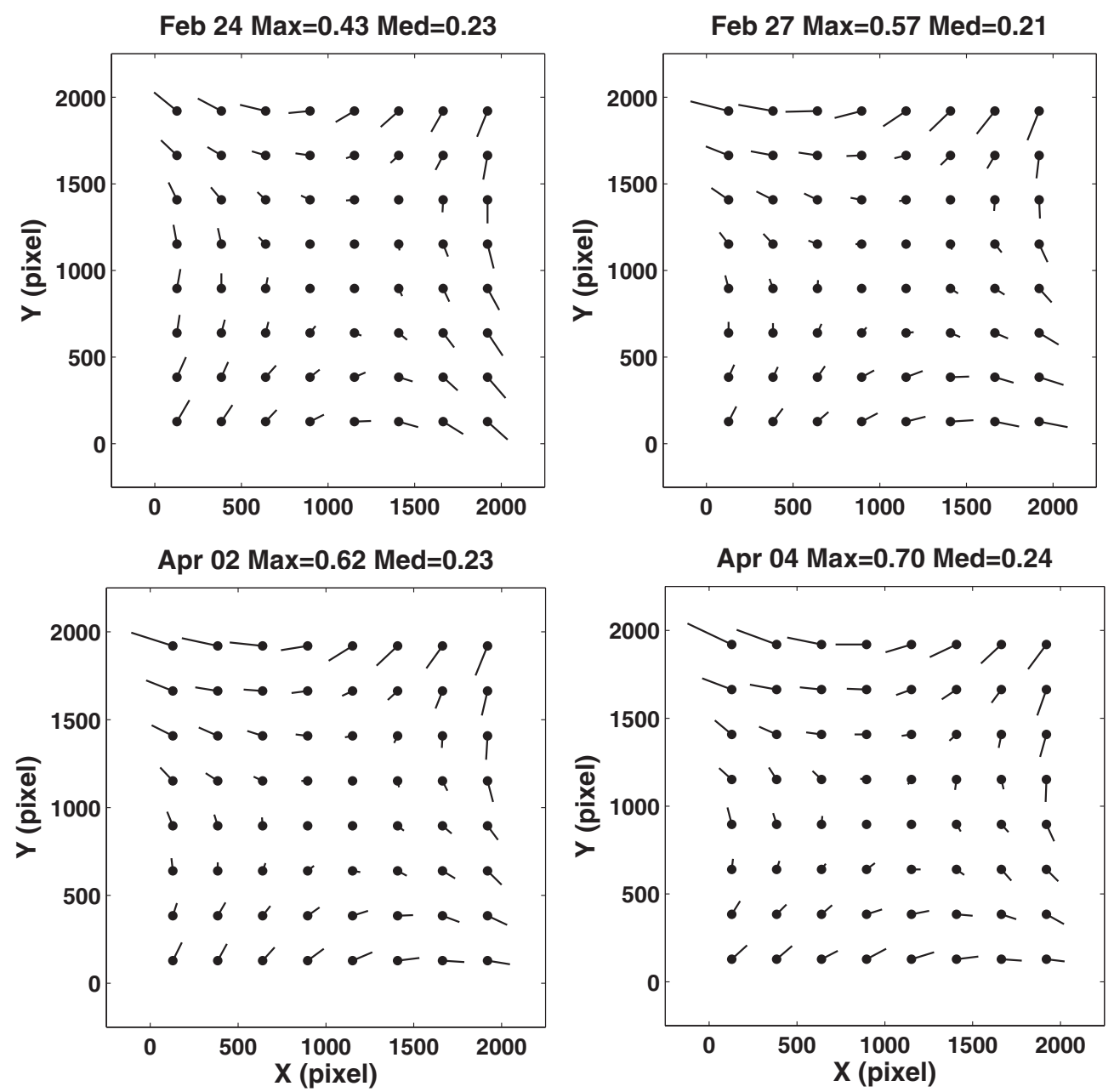

Figure 4. GDs derived from a Johnson I filter from observations of M35 and M67 on four nights. At the top of each panel, the maximum GD and the median GD of each night are also listed in units of pixels. A factor of 400 is used to exaggerate the magnitude of each GD vector.

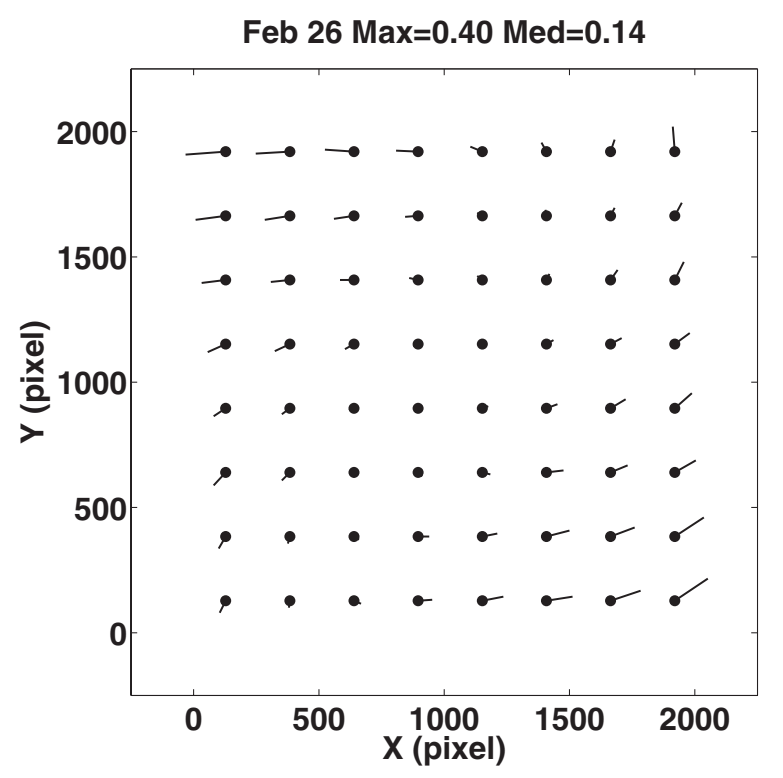

Figure 5. Smaller but visible swirl-like GD is also found while no filter is used based on the observations on the night of February 26. At the top of the panel, the maximum GD and the median GD are also listed in units of pixels. A factor of 400 is used to exaggerate the magnitude of each GD vector.
$(\mathrm{O}-\mathrm{C})$ positional residuals for $\mathrm{M} 35$ become much better after GD correction for most brighter stars. For fainter stars, there is only small improvement owing to their low signal noise ratios.

\subsection{Positional Measurement of Phoebe}

Usually, only a few stars appear in a CCD frame for Phoebe. Figure 8 is a typical CCD frame for Phoebe in which only three stars (with circles) can be found in the UCAC2 catalog (Zacharias et al. 2004). Therefore, we cannot deliver the GD pattern based only on these CCD frames. A simple practice is to adopt the mean GD solution delivered from some open cluster in the same night and with the same filter to remove the effect of GD on the pixel positions of the reference stars and Phoebe. Then a classical reduction is performed using Equations (2) and (3) to derive the positional residual of Phoebe. In more detail, we first remove the GD effect using a bilinear interpolation from the original and numerical GD distribution. Then, we compare the corrected pixel positions for these reference stars with their standard coordinates to derive transformation parameters by least squares for each field of view. Specifically, all astrometric effects are taken into account in the computation, i.e., the atmospheric refraction is incorporated into the topocentric positions before central projection. In order to 


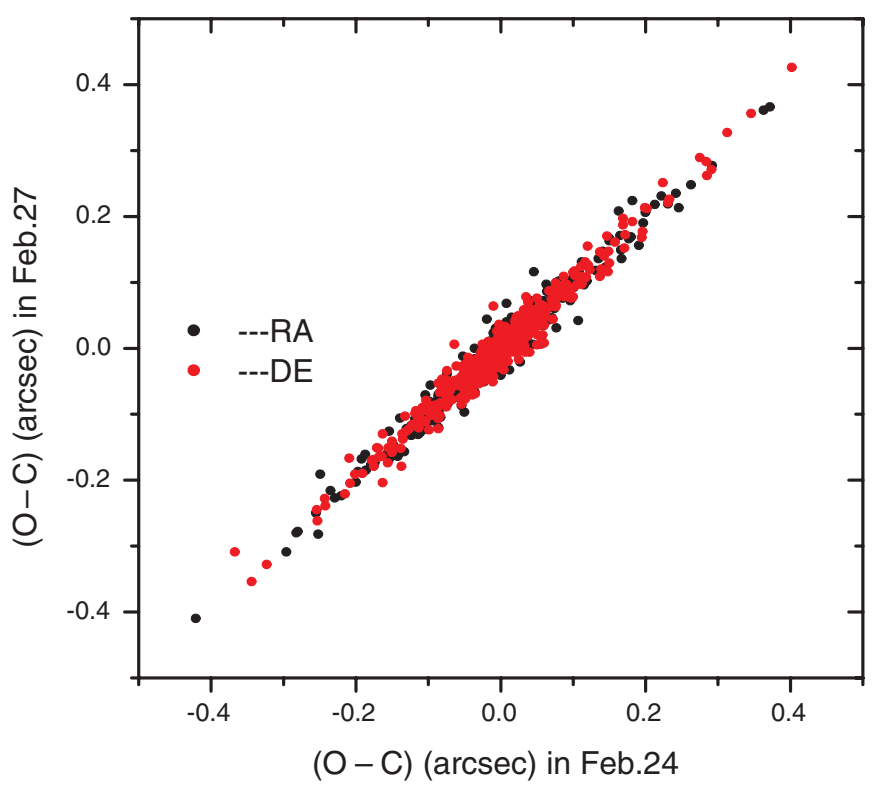

Figure 6. $(\mathrm{O}-\mathrm{C})$ residuals on two nights.

compare the effect of GD on the positional measurement of Phoebe, we use a modern ephemeris developed by the IMCCE via their Web site http://www.imcce.fr/. DE405, developed by JPL (Standish 1998), is adopted for the primary planet Saturn.

To illustrate the effect of GD correction, the results without GD corrections are also obtained under the condition that all other computations are completely the same. Figure 9 shows the $(\mathrm{O}-\mathrm{C})$ residuals of Phoebe. On the whole, the dispersion after GD correction becomes smaller. The most obvious improvement of the $(\mathrm{O}-\mathrm{C})$ residuals appears in the second line of panels in Figure 9. Specifically, the dispersion of the $(\mathrm{O}-\mathrm{C})$ residuals in right ascension becomes much smaller in the second and third nights of the April campaign. Another obvious example is in the declination in the second night of the April campaign, where the mean $(\mathrm{O}-\mathrm{C})$ changed significantly, which can be explained by two factors, that is to say, the positional refinement of calibration reference stars due to GD correction works together with the positional refinement of Phoebe in the same direction, causing the combined results to be much better. A slightly greater dispersion in the $(\mathrm{O}-\mathrm{C})$ residuals appears on the third night of the April campaign by using the $R$ filter in declination,

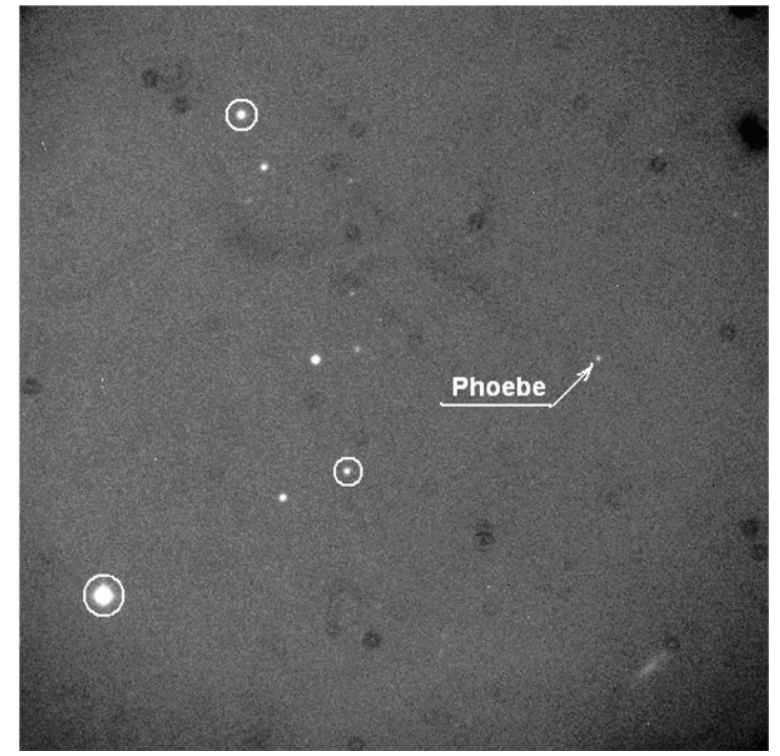

Figure 8. Typical CCD frame for the satellite Phoebe taken on February. Only three reference stars (with circles) can be found in UCAC2.

which deserves to be studied in the future. In addition, Figure 10 shows the $(\mathrm{O}-\mathrm{C})$ residuals in terms of the pixel coordinates in the CCD frame before and after GD correction on two nights of observations (April 2 and 3). The changes are quite obvious.

In addition, statistics are listed in Table 4 for the observations of Phoebe and all results before and after GD corrections are shown. It is clear that a significant improvement appears in the positional residuals of Phoebe. In addition, there are some systematic trends appearing in Figure 9 for the $(\mathrm{O}-\mathrm{C})$ residuals in both directions. This suggests that the ephemeris of Phoebe or DE405 for Saturn might need to be refined. Further observations are certainly required.

\section{DISCUSSIONS}

Our procedure is different from the operation by Anderson \& King (2003), where no central projection is done and a four-parameter linear transformation is performed for the pixel positions between two different CCD frames. We take the central projection into account, and then the four-parameter
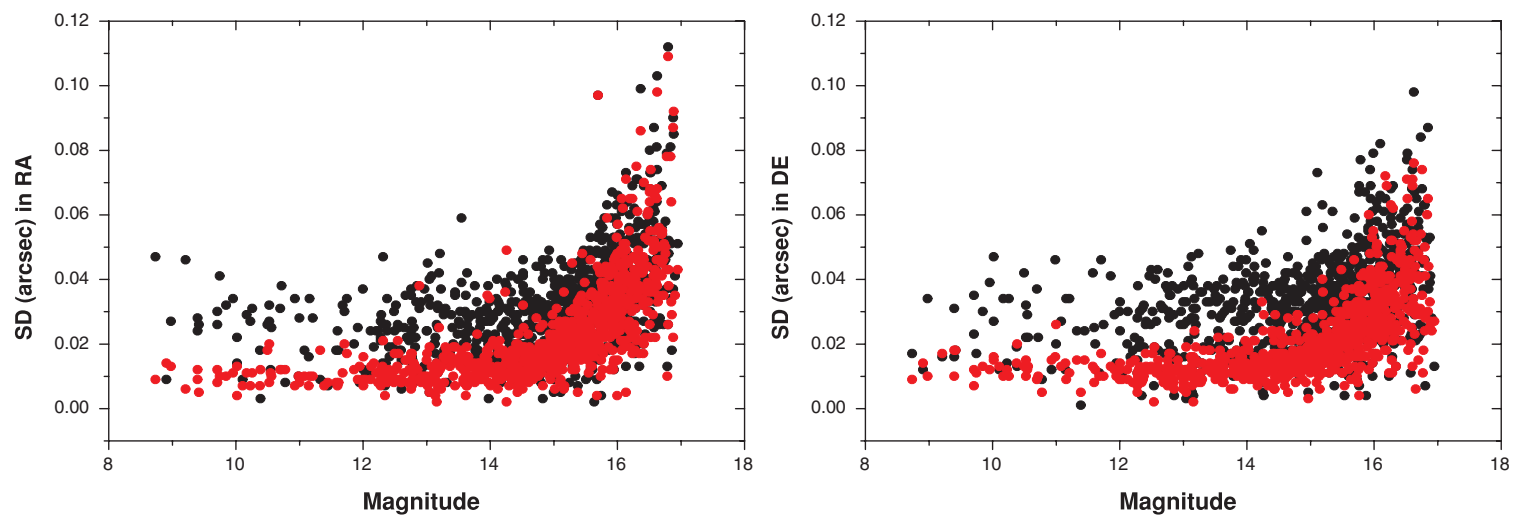

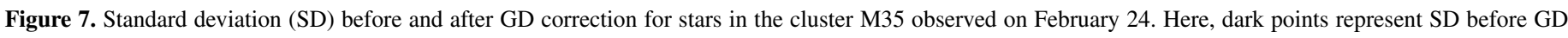
correction and red ones represent SD after GD correction. 
Table 3

Mean Geometric Distortions in $x$ - and $y$-coordinate Axes and Their Standard Deviations in Units of Pixels Derived from $I$ and $R$ filters, Respectively

\begin{tabular}{|c|c|c|c|c|c|c|c|c|c|}
\hline & $y$ & $d x_{R}$ & $\sigma_{x}$ & $d y_{R}$ & $\sigma_{y}$ & $d x_{I}$ & $\sigma_{x}$ & $d y_{I}$ & $\sigma_{y}$ \\
\hline 128 & 128 & -0.46 & 0.05 & -0.16 & 0.05 & 0.17 & 0.05 & 0.25 & \\
\hline 384 & 128 & -0.45 & 0.05 & -0.04 & 0.05 & 0.17 & 0.04 & 0.21 & \\
\hline 640 & 128 & -0.40 & 0.03 & 0.06 & .07 & 19 & & & \\
\hline 896 & 128 & -0.33 & 0.03 & 0.18 & & 24 & & 0.14 & \\
\hline 1152 & 128 & -0.26 & 0.02 & 0.28 & 0.06 & 0.28 & & 0.09 & 0.0 \\
\hline 1408 & 128 & -0.20 & 0.01 & 0.39 & 0.06 & 0.31 & 0.02 & 0.02 & \\
\hline 1664 & 128 & -0.16 & 0.03 & 0.47 & 0.08 & 0.31 & 0.02 & -0.06 & \\
\hline 1920 & 128 & & 0.04 & 0.68 & 0.08 & 0.30 & 0.06 & -0.09 & \\
\hline 128 & 384 & -0.39 & 0.04 & -0.24 & 0.07 & 11 & & & \\
\hline 384 & 384 & -0.34 & 0.03 & -0.11 & 0.04 & 11 & & 0.17 & 0.0 \\
\hline 640 & 384 & -0.31 & 0.02 & 0.02 & 0.04 & 0.14 & 0.02 & 0.15 & 0.0 \\
\hline 896 & 384 & & 0.01 & 0.12 & & 0.18 & .03 & 0.10 & \\
\hline 1152 & 384 & & 0.01 & & & & 0.03 & 0.07 & 0.0 \\
\hline 1408 & 384 & & & & & & 0.03 & -0.01 & \\
\hline 1664 & 384 & & & .44 & & & & -0.10 & 0.0 \\
\hline 1920 & 384 & -0.02 & 0.02 & 0.62 & & 26 & 0.0 & -0.16 & 0.0 \\
\hline 128 & 640 & & 0.03 & & & 02 & & & \\
\hline 384 & 640 & & & & & & & & \\
\hline 640 & 640 & & & & & & & & \\
\hline 896 & 640 & -0 . & & 0.06 & & & & & 0.02 \\
\hline 1152 & 640 & -0.13 & 0.01 & 0.17 & 0.0 & 11 & 0.01 & -0.01 & 0.02 \\
\hline 140 & 640 & -0.05 & 0.02 & 0.27 & 0.02 & 0.12 & 0.01 & -0.07 & \\
\hline 1664 & 640 & & & 35 & & 15 & 0.03 & & \\
\hline 1920 & 64 & & & & & & & & \\
\hline 128 & 896 & -0.24 & 0.02 & -0.36 & & -0.03 & & & 0.0 \\
\hline 384 & 896 & -0.20 & 0.02 & -0.22 & 0 & -0.03 & 0. & 0.17 & 0.0 \\
\hline 640 & 896 & -0.14 & 0.02 & -0.12 & & & & & \\
\hline 896 & 896 & -0.08 & 0.01 & 0.01 & & & 0.0 & & \\
\hline 1152 & 89 & & & & & & & & \\
\hline 140 & 89 & & 0. & & & & & & \\
\hline 166 & 89 & & 0.0 & & & & 0.0 & -0.13 & 0.0 \\
\hline 1920 & 896 & & & & & & & -0.23 & \\
\hline 128 & 1152 & -0.17 & 0.03 & -0.40 & 0.03 & -0.12 & 0.05 & 0.20 & \\
\hline 384 & 52 & & 0.02 & 32 & & & & & \\
\hline & & & & & & & & & \\
\hline 896 & 1 & -0.02 & 0.0 & -0.08 & & -0 & 0.0 & & 0.0 \\
\hline 1152 & & & & & & -0.03 & 0.01 & -0 & \\
\hline 1408 & & & & & & & 0.02 & -0.11 & \\
\hline 1664 & & & & & & & 0.03 & -0.15 & \\
\hline & & & & & & & & & \\
\hline 128 & 1408 & -0. & 0.0 & -0.49 & & -0 & & & \\
\hline 384 & 1408 & 0.08 & 0.02 & 0.33 & 04 & 22 & 0.0 & 0.14 & 0.0 \\
\hline 64 & & -0.01 & & & & & & & \\
\hline 896 & 1408 & & & & & & 0. & & \\
\hline 1152 & & & & & & & & & \\
\hline 1408 & 140 & & & & & & 0.0 & 9 & \\
\hline 1664 & 1408 & & 0.0 & & 0.02 & -0.03 & 0.02 & -0.18 & 0.0 \\
\hline 1920 & 1408 & & & & & -0.05 & 0.05 & -0.31 & \\
\hline 128 & 1664 & -0.08 & & & 0.02 & -0.35 & 0.07 & 0.17 & 0.0 \\
\hline 384 & & & & & & & & & \\
\hline 640 & 1664 & & & & & & & & \\
\hline 896 & 1664 & & & 9 & 0.03 & -0.23 & 0.03 & -0.01 & \\
\hline 115 & & & & & & -0 & 0.04 & -0.07 & \\
\hline 1408 & 1664 & & 0.02 & & 0.02 & -0.15 & 0.03 & -0.14 & 0.0 \\
\hline 1664 & 1664 & & 0.02 & & .02 & & 0.03 & 0.22 & 0.0 \\
\hline 1920 & & & & & & & & & \\
\hline 128 & 1920 & -0.0 & & & & & & 0.25 & \\
\hline 38 & & & & & & & & 0.16 & \\
\hline 640 & 1920 & & & & 0.04 & & 0.04 & 0.06 & 0.0 \\
\hline 896 & 1920 & & 0.02 & & 0.04 & -0.38 & 0.04 & -0.04 & 0.0 \\
\hline 1152 & 1920 & & 0.03 & & & & 0.03 & & \\
\hline 1408 & 1920 & & 0.05 & -0.02 & 0.05 & & 0.04 & & \\
\hline & & & & & & & 0.04 & -0.31 & \\
\hline 1920 & 1920 & 0.77 & 0.12 & 0.36 & 0.07 & -0.20 & 0.05 & -0.39 & 0.0 \\
\hline
\end{tabular}

Notes. The first two columns are the pixel locations of the grid points in the CCD coordinate system. Columns 3 and 5 are the mean GD along the $x$ - and $y$-coordinate axes, respectively, at the grid points $(x, y)$ when the Johnson $R$ filter is used on four nights. Columns 4 and 6 are the standard deviations of the corresponding GD in the $x$ - and $y$-coordinate directions, respectively. Similarly, Columns 7 and 9 are the mean GD in $x$ - and $y$-coordinate axes for the Johnson $I$ filter, and Columns 8 and 10 are their respective standard deviations derived from six nights of separate observations.
Table 4

Statistics of $(\mathrm{O}-\mathrm{C})$ Residuals of Phoebe Before and After GD Correction for Each Data Set

\begin{tabular}{|c|c|c|c|c|c|c|}
\hline ID & No. and Filter & & $\mu_{\alpha}$ & $\sigma_{\alpha}$ & $\mu_{\delta}$ & $\sigma_{\delta}$ \\
\hline 0224 & $10 \mathrm{I}$ & $\begin{array}{c}\text { before } \\
\text { after }\end{array}$ & $\begin{array}{l}0.074 \\
0.077\end{array}$ & $\begin{array}{l}0.045 \\
0.036\end{array}$ & $\begin{array}{l}-0.077 \\
-0.097\end{array}$ & $\begin{array}{l}0.107 \\
0.021\end{array}$ \\
\hline 0225 & $19 N$ & $\begin{array}{c}\text { before } \\
\text { after }\end{array}$ & $\begin{array}{l}-0.020 \\
-0.033\end{array}$ & $\begin{array}{l}0.033 \\
0.032\end{array}$ & $\begin{array}{l}0.118 \\
0.098\end{array}$ & $\begin{array}{l}0.063 \\
0.059\end{array}$ \\
\hline 0226 & $21 N$ & $\begin{array}{c}\text { before } \\
\text { after }\end{array}$ & $\begin{array}{l}-0.074 \\
-0.074\end{array}$ & $\begin{array}{l}0.040 \\
0.040\end{array}$ & $\begin{array}{l}0.039 \\
0.043\end{array}$ & $\begin{array}{l}0.023 \\
0.023 \\
\end{array}$ \\
\hline 0227 & $15 \mathrm{I}$ & $\begin{array}{c}\text { before } \\
\text { after }\end{array}$ & $\begin{array}{l}-0.018 \\
-0.019 \\
\end{array}$ & $\begin{array}{l}0.075 \\
0.042 \\
\end{array}$ & $\begin{array}{l}0.000 \\
0.009 \\
\end{array}$ & $\begin{array}{l}0.029 \\
0.021 \\
\end{array}$ \\
\hline 0401 & $19 \mathrm{I}$ & $\begin{array}{c}\text { before } \\
\text { after }\end{array}$ & $\begin{array}{l}0.097 \\
0.106\end{array}$ & $\begin{array}{l}0.033 \\
0.033\end{array}$ & $\begin{array}{l}0.103 \\
0.096 \\
\end{array}$ & $\begin{array}{l}0.019 \\
0.019\end{array}$ \\
\hline 0402 & $28 \mathrm{R}$ & $\begin{array}{c}\text { before } \\
\text { after }\end{array}$ & $\begin{array}{l}0.139 \\
0.166 \\
\end{array}$ & $\begin{array}{l}0.044 \\
0.017 \\
\end{array}$ & $\begin{array}{r}-0.099 \\
0.082 \\
\end{array}$ & $\begin{array}{l}0.029 \\
0.029\end{array}$ \\
\hline 0403 & $55 \mathrm{I}$ & $\begin{array}{c}\text { before } \\
\text { after }\end{array}$ & $\begin{array}{l}0.201 \\
0.197\end{array}$ & $\begin{array}{l}0.105 \\
0.058\end{array}$ & $\begin{array}{l}0.022 \\
0.022\end{array}$ & $\begin{array}{l}0.032 \\
0.028\end{array}$ \\
\hline 0403 & $23 R$ & $\begin{array}{c}\text { before } \\
\text { after }\end{array}$ & $\begin{array}{l}0.232 \\
0.196\end{array}$ & $\begin{array}{l}0.028 \\
0.020\end{array}$ & $\begin{array}{l}-0.028 \\
-0.010\end{array}$ & $\begin{array}{l}0.020 \\
0.025\end{array}$ \\
\hline 0404 & $30 I$ & $\begin{array}{c}\text { before } \\
\text { after }\end{array}$ & $\begin{array}{l}0.178 \\
0.151\end{array}$ & $\begin{array}{l}0.038 \\
0.030\end{array}$ & $\begin{array}{l}0.024 \\
0.037\end{array}$ & $\begin{array}{l}0.055 \\
0.028\end{array}$ \\
\hline
\end{tabular}

Notes. Columns 1 and 2 are the data ID and the number of observations with the filter used, respectively, on each night. The third column ("before" or "after") shows the statistics in both directions before or after GD correction. The following two columns list the mean $(\mathrm{O}-\mathrm{C})\left(\mu_{\alpha}\right)$ and its standard deviation $\left(\sigma_{\alpha}\right)$ in right ascension. The last two columns list the mean $(\mathrm{O}-\mathrm{C})\left(\mu_{\delta}\right)$ and its standard deviation $\left(\sigma_{\delta}\right)$ in declination. All units are in arcseconds.

linear transformation is performed only in the same CCD frame. There are three advantages to our proposed approach. First, it uses the information of an astrometric catalog, which is relatively precise and stable. Thus, we need not assume some zero coefficients, as was the practice of Anderson \& King (2003) because of an unknown absolute scale factor. Second, it allows us to compute some astrometric effects (for example, atmospheric refraction) very conveniently to ensure a fourparameter linear transformation (instead of a six-parameter one). Last, it is easier to understand for a classical photographing astrometrist.

A four-parameter linear transformation is more advantageous since only two reference stars are needed to calibrate the CCD field of view after GD correction. It is preferable for a faint movable target (such as Phoebe) where only a few reference stars can be found in some catalogs. Of course, careful attention must be paid to the positional computation of the working stars (those to be used to derive the GD) and the pixel of the CCD chip must be square. Specifically, all astrometric effects must be taken into account, such as diurnal aberration, atmospheric refraction, and central projection. Due to these reasons, we prefer to have initial catalog positions for these working stars. At present, there are several astrometric catalogs available, such as PPMXL, USNO-B1, and others. According to our findings, GD patterns depend on the filter used, since a filter can distort the direction of light passing through it (detailed formulae and discussions can be found in van Altena \& Monnier 1968). Because of this, better quality filter should be manufactured. More observations are needed to study the law of variation of the GD pattern, especially when the telescope is operated in different pointings and under different environmental conditions such as temperature and pressure. 

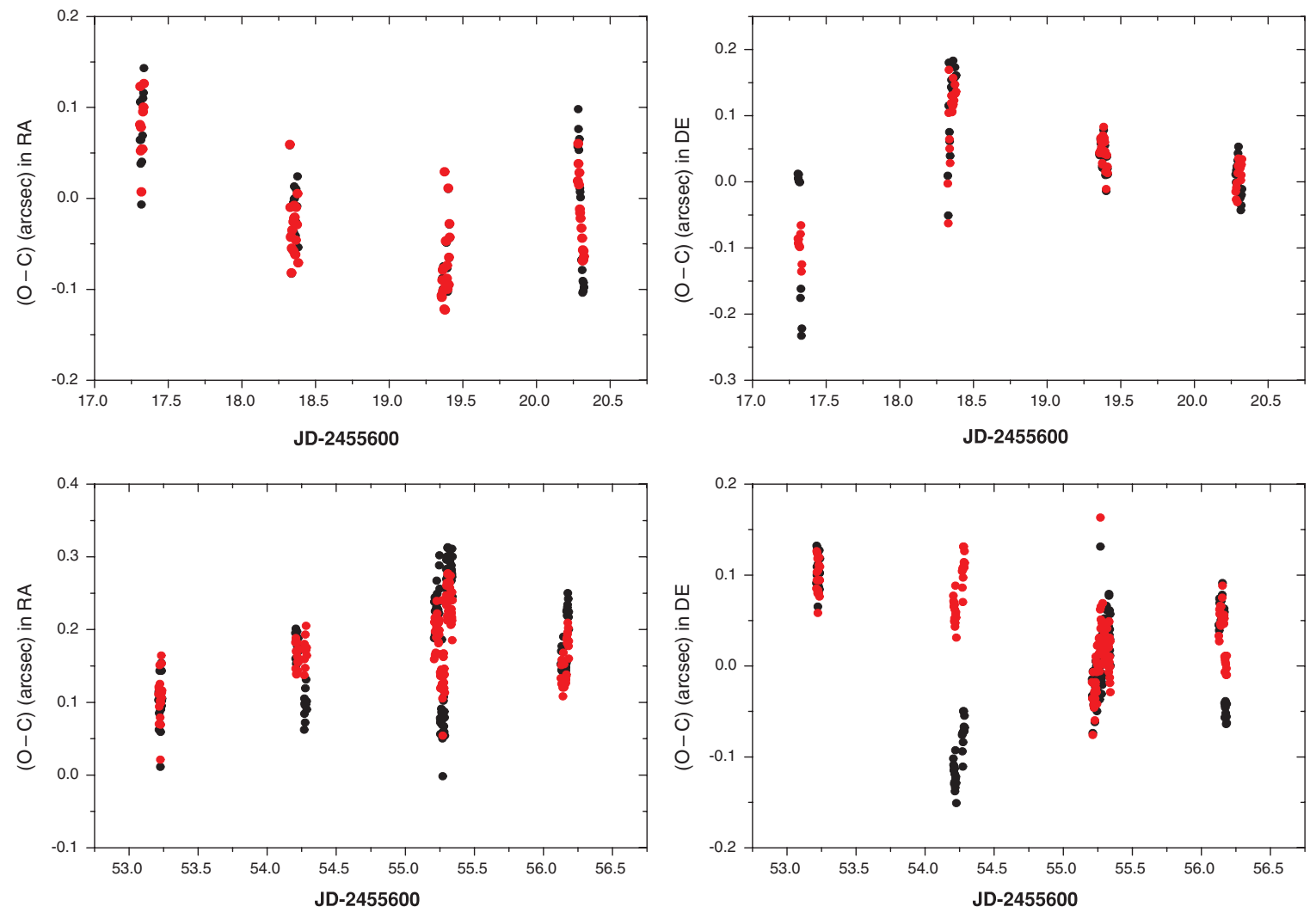

Figure 9. $(\mathrm{O}-\mathrm{C})$ residuals of the positions of Phoebe in two campaigns. The upper two panels are for the campaign in February and the bottom two panels are for that in April. The dark points represent the $(\mathrm{O}-\mathrm{C})$ before GD correction and the red ones represent the $(\mathrm{O}-\mathrm{C})$ after GD correction.
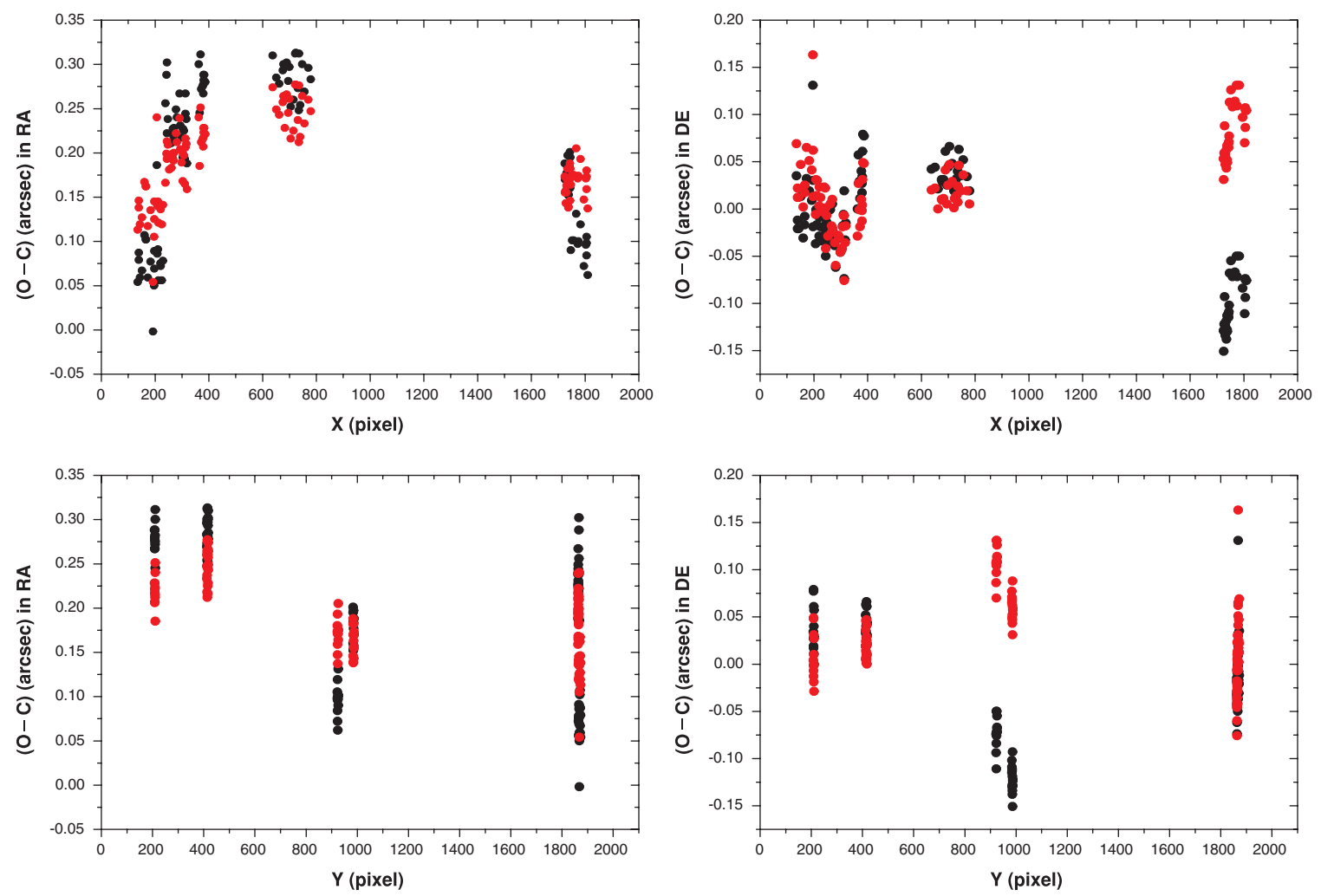

Figure 10. $(\mathrm{O}-\mathrm{C})$ residuals of positions of Phoebe on April 2 and 3. The upper two panels show the $(\mathrm{O}-\mathrm{C})$ residuals with respect to the $x$-coordinate of the $\mathrm{CCD}$ chip. The bottom two panels are the $(\mathrm{O}-\mathrm{C})$ residuals with respect to the $y$-coordinate of the CCD chip. The dark points represent the $(\mathrm{O}-\mathrm{C})$ residuals before $\mathrm{GD}$ correction and the red ones represent the $(\mathrm{O}-\mathrm{C})$ residuals after $\mathrm{GD}$ correction. 


\section{CONCLUSIONS}

A convenient but effective approach is proposed to solve the GD of a CCD field of view. After many CCD frames of some open clusters observed by a $1 \mathrm{~m}$ telescope at the Yunnan Observatory are used to test the approach, its effectiveness was verified, especially its application to the observations of Phoebe, the ninth satellite of Saturn. Positional precision for both stars from clusters and the satellite Phoebe are found to be improved significantly after correcting the GD. It is also found that the GD depends strongly on the filter used. In addition, even when no filter is used a small but obvious-enough distortion appears as well.

This research work is financially supported by the National Natural Science Foundation of China (grant Nos. 10973007, 11273014,11150110138 ) and partly by the Fundamental Research Funds for the Central Universities. The authors are grateful to the $1 \mathrm{~m}$ telescope working staff, especially to chief scientist Dr. Qian Sheng-Bang for their long-term support and help. Valuable suggestions from the anonymous referee make the explanations of the proposed approach much clearer. In addition, the discussions with Professor Mao Wei at Yunnan Observatory were constructive.

\section{REFERENCES}

Anderson, J., Bedin, L. R., Piotto, G., et al. 2006, A\&A, 454, 1029

Anderson, J., \& King, R. K. 2003, PASP, 115, 113

Bellini, A., \& Bedin, L. R. 2010, A\&A, 517, A34

Colas, F., \& Arlot, J. E. 1991, A\&A, 252, 402

French, R. G., McGhee, C. A., Frey, M., et al. 2006, PASP, 118 246

Green, R. M. 1985, Spherical Astronomy (Cambridge: Cambridge Univ. Press) Harper, D., Murray, C. D., Beurle, K., et al. 1997, A\&AS, 121, 65

Peng, Q. Y., He, H. F., Lainey, V., \& Vienne, A. 2012, MNRAS, 419, 1977

Peng, Q. Y., Vienne, A., Han, Y. B., \& Li, Z. L. 2004, A\&A, 424, 339

Peng, Q. Y., \& Zhang, Q. F. 2006, MNRAS, 366, 208

Poulet, F., \& Sicardy, B. 2001, A\&A, 366, 339

Ren, J. J., \& Peng, Q. Y. 2010, Astron. Res. Tech., 7, 115

Roeser, S., Demleitner, M., \& Schilbach, E. 2010, AJ, 139, 2440

Shen, K. X., Dourneau, G., Qiao, R. C., \& Liu, J. R. 2001, A\&A, 367, 1061

Standish, E. M., Jr. 1998, JPL Planetary and Lunar Ephemeris, DE405/LE405 JPL Interoffice Memo. 321-98-048.0. JPL, Pasadena

van Altena, W. F., \& Monnier, R. C. 1968, AJ, 73, 649

van der Marel, R. P., Anderson, J., Cox, C., et al. 2007, Instrument Science Report ACS 2007-07

Vienne, A., Thuillot, W., Veiga, C. H., et al. 2001, A\&A, 380, 727

Yadav, R. K. S., Bedin, L. R., Piotto, G., et al. 2008, A\&A, 484, 609

Zacharias, N., Urban, S. E., Zacharias, M. I., et al. 2004, AJ, 127,3043 